Ferrata Storti Foundation

\title{
Murine tissue factor disulfide mutation causes a bleeding phenotype with sex specific organ pathology and lethality
}

Haematologica 2020

Volume 105(10):2484-2495

\section{Correspondence:}

FELIX C. TANNER

felix.tanner@usz.ch

Received: February 7, 2019.

Accepted: August 30, 2019.

Pre-published: September 5, 2019.

doi:10.3324/haematol.2019.218818

(C)2020 Ferrata Storti Foundation

Material published in Haematologica is covered by copyright. All rights are reserved to the Ferrata Storti Foundation. Use of published material is allowed under the following terms and conditions:

https://creativecommons.org/licenses/by-nc/4.0/legalcode. Copies of published material are allowed for personal or internal use. Sharing published material for non-commercial purposes is subject to the following conditions:

https://creativecommons.org/licenses/by-nc/4.0/legalcode, sect. 3. Reproducing and sharing published material for commercial purposes is not allowed without permission in writing from the publisher.

\section{Susanna H. M. Sluka, ${ }^{1 *}$ Simon F. Stämpfli ${ }^{1,2,3^{*}}$ Alexander Akhmedov, ${ }^{1}$ Tanja Klein Rodewald, ${ }^{4}$ Adrián Sanz-Moreno, ${ }^{4}$ Marion Horsch, ${ }^{4}$ Paula Grest, ${ }^{5}$ Andrea S. Rothmeier, ${ }^{6}$ Birgit Rathkolb, ${ }^{4,7,8}$ Anja Schrewe, ${ }^{4}$ Johannes Beckers, ${ }^{4,8,9}$ Frauke Neff, ${ }^{4}$ Eckhard Wolf, ${ }^{7}$ Giovanni G. Camici, ${ }^{1}$ Helmut Fuchs, ${ }^{4}$ Valerie Gailus Durner, ${ }^{4}$ Martin Hrabě de Angelis, ${ }^{4,8,9}$ Thomas F. Lüscher, ${ }^{1,2}$ Wolfram Ruf ${ }^{6,10}$ and Felix C. Tanner ${ }^{1,2}$}

\begin{abstract}
*SHMS and SFS contributed equally as co-first authors
${ }^{1}$ Center for Molecular Cardiology, University of Zurich, Zurich, Switzerland; ${ }^{2}$ Department of Cardiology, University Heart Center, University Hospital, Zurich, Switzerland; ${ }^{3}$ Cardiology Division, Heart Center, Luzerner Kantonsspital, Luzern, Switzerland; ${ }^{4}$ German Mouse Clinic, Institute of Experimental Genetics, Helmholtz Zentrum München and German Research Center for Environmental Health, Neuherberg, Germany; ${ }^{5}$ Institute of Veterinary Pathology, University of Zurich, Zurich, Switzerland; ${ }^{6}$ Department of Immunology and Microbiology, Scripps Research, La Jolla, CA, USA; 'Institute of Molecular Animal Breeding and Biotechnology, Gene Center, Ludwig-Maximilians-University München, Munich, Germany; ${ }^{8}$ German Center for Diabetes Research (DZD), Neuherberg, Germany; ${ }^{9}$ Experimental Genetics, School of Life Science Weihenstephan, Technische Universität München, Freising, Germany and ${ }^{10} \mathrm{Center}$ for Thrombosis and Hemostasis Johannes Gutenberg University Medical Center, Mainz, Germany
\end{abstract}

\section{ABSTRACT}

issue factor (TF) is highly expressed in sub-endothelial tissue. The extracellular allosteric disulfide bond Cys186-Cys209 of human TF shows high evolutionary conservation and in vitro evidence suggests that it significantly contributes to TF procoagulant activity. To investigate the role of this allosteric disulfide bond in vivo, we generated a C213G mutant Tf mouse by replacing Cys213 of the corresponding disulfide Cys190-Cys213 in murine Tf. A bleeding phenotype was prominent in homozygous C213G Tf mice. Pre-natal lethality of one third of homozygous offspring was observed between embryonic (E) day E9.5 and E14.5 associated with placental hemorrhages. After birth, homozygous mice suffered from bleedings in different organs and reduced survival. Homozygous C213G Tf male mice showed higher incidence of lung bleedings and lower survival rates than females. In both sexes, C213G mutation evoked a reduced protein expression (about 10fold) and severely reduced pro-coagulant activity (at least 100-fold). Protein glycosylation was impaired and cell membrane exposure decreased in macrophages in vivo. Single housing of homozygous C213G Tf males reduced the occurrence of severe bleeding and significantly improved survival, suggesting that inter-male aggressiveness might significantly account for the sex differences. These experiments show that the TF allosteric disulfide bond is of crucial importance for normal in vivo expression, post-translational processing and activity of murine TF. Although C213G Tf mice do not display the severe embryonic lethality of $T f$ knock-out mice, their postnatal bleeding phenotype emphasizes the importance of fully functional TF for hemostasis.

\section{Introduction}

Tissue factor (TF) is the cellular activator of the extrinsic pathway of blood coagulation. It is expressed in the sub-endothelial wall of blood vessels and organ parenchyma, leading to activation of coagulation after vessel injury. Levels of TF 
expression, however, differ significantly between organs: heart, brain, lung, and uterus exhibit very high TF expression, while TF is barely detectable in skeletal muscle and joints. ${ }^{1,2}$ Total $T f$ deletion in mice leads to embryonic death due to insufficient development of yolk sac vessels and vascular failure..$^{3-5}$ A TF transgene inducing low expression of human full length TF (flTF) in mice (low-TF mice, 1\% of normal murine TF levels) is sufficient to rescue Tf knock-out (KO) mice from embryonic lethality ${ }^{6}$ but not to restore normal postnatal hemostatic function. These mice suffer from spontaneous hemorrhages particularly in organs which in wild-type (wt) mice express high levels of TF such as the heart, lung, brain, gastrointestinal tract, and testis.-9 Fatal lung hemorrhages are very common in low-TF mice ${ }^{8,10}$ and are further enhanced when exposed to either intratracheal lipopolysaccharide ${ }^{11}$ or infected with influenza A virus. ${ }^{12}$ Similarly, fatal brain hemorrhages have been observed in low-TF mice. ${ }^{10}$ Furthermore, female low- $T F$ mice exhibit fatal hemorrhages in the placenta and uterus during pregnancy and post partum, suggesting that TF is crucial for uterine hemostasis. ${ }^{13}$ In line with these observations, tail bleeding time in low-TF mice was increased. ${ }^{14}$

Due to its sub-endothelial localization, TF is separated from circulating clotting factors in the absence of an injury. Minor amounts of TF are however expressed on the surface of monocytes and come in contact with blood. ${ }^{15,16}$ To control pro-coagulant activity, TF activity is post-translationally suppressed by a mechanism called encryption. ${ }^{17}$ The mechanism of encryption is controversially discussed: several studies suppose that predominantly exposure of negatively charged phospholipids account for the decryption of TF activity, ${ }^{18,19}$ while others suggest activation via thiol disulfide exchange reactions ${ }^{20-25}$ involving complement activation. Human TF indeed contains two disulfide bonds (Cys49-Cys57 and Cys186-Cys209) which are conserved in mice (Cys47-Cys55 and Cys190-Cys213) and many other species. The C-terminal bond shares sequence features of other allosteric disulfide bonds and is important for TF pro-coagulant activity ${ }^{26}$ and thus crucial for regulation of TF activity in cell cultures. ${ }^{27,28}$ Release of procoagulant, TF-containing extracellular vesicles (EV) from bone marrow-derived macrophages (BMDM) and smooth muscle cells was shown to be regulated by ATP stimulation of the purinergic receptor $\mathrm{P} 2 \mathrm{X} 7,{ }^{29}$ also depending on thiol-exchange reactions. ${ }^{29}$

We generated a C213G Tf knock-in (KI) mouse model to investigate the function of disulfide bond mutated TF in vivo and its contribution to hemostasis.

\section{Methods}

\section{Construction of the C213G Tf targeting vector}

The coding sequence of the murine full-length $T f$ isoform containing a $\mathrm{T721G}$ mutation as well as the flanking genomic regions were inserted into KpnI/NotI restriction sites of a LNTK vector to replace the genomic TF locus. Embryonic stem cell transfection (129P2/OlaHsd) and blastocyst injection was conducted by PolyGene (Ruemlang, Switzerland). Chimeric mice were bred with C57BL/6 mice.

\section{Animals}

One mouse colony was bred of heterozygous animals on a mixed 129P2/OlaHsd-C57BL/6 background (50\% from each strain). Mice were backcrossed to C57BL/6 (B6). All tests performed were approved by the responsible authority of the local government.

\section{Embryonic analysis}

For embryonic analyses heterozygous C213G/+ Tf mice were bred and females were controlled for plugs every morning (day of the plugs considered E0.5).

\section{Pathological analysis}

All organs were fixed in $4 \%$ buffered formalin and embedded in paraffin for histological examination. Four- $\mu$ m-thick sections were cut and stained.

\section{Expression profiling}

Total RNA was isolated from organs just before microarray hybridization. Amplified RNA was hybridized and analyzed on Illumina MouseRef8 v2.0 Expression Bead Chips containing about 25,000 probes.

\section{Bleeding time}

After onset of anesthesia, tails were pre-warmed in a $37^{\circ} \mathrm{C}$ water bath for $10 \mathrm{~min}$. Then $0.5 \mathrm{~mm}$ of the tail tip was amputated, and the tail was immediately put back to $37^{\circ} \mathrm{C}$ PBS.

\section{Tail-cuff blood pressure measurement}

Blood pressure was measured in non-anesthetized mice with a non-invasive tail-cuff method using the MC4000 Blood Pressure Analysis Systems.

\section{TF expression analysis}

RNA was extracted using TRIzol Reagent (Molecular Research Center). Transcript levels were quantified by Real Time PCR using SyBr Green Master Mix on an Applied Biosystems 7300 System. Protein was extracted by grinding of organ tissue in lysis buffer. Samples were separated by $10 \%$ SDS-PAGE and transferred to a PVDF membrane (Immobilion ${ }^{\circledR}$-FL, Millipore). The membrane was stained with anti-mouse TF rabbit antiserum $(\mathrm{R} 8084)^{29}$ and anti-human GAPDH (cross-reactive to mouse) mouse monoclonal (MAB374, Millipore) primary antibodies, followed by secondary antibodies.

\section{TF activity}

Tissue was lysed in HEPES-saline containing $0.02 \%$ sodium azide (HBS) and diluted in HBS containing $1 \mathrm{mg} / \mathrm{mL} \mathrm{BSA}$ and 50 $\mu \mathrm{M}$ phospholipid vesicles to measure TF activity using a plasma clotting assay.

\section{Macrophage experiments}

Femora and tibiae were dissected and flushed with RPMI. Cells were resuspended in 90\% FCS, 10\% DMSO. BMDM were generated from total BM cultures as described previously. ${ }^{30}$ Cell surface TF activity was determined with $0.5 \mathrm{nM}$ factor VIIa (FVIIa) and $50 \mathrm{nM}$ factor $\mathrm{X}(\mathrm{FX})$ in $\mathrm{HBS}$ by measuring a time course of FXa generation. TF EV activity was measured in HBS with 2 nM FVIIa and $100 \mathrm{nM} \mathrm{FX}$. EV prothrombinase activity was measured in HBS with $10 \mathrm{nM} \mathrm{FVa,} 5 \mathrm{nM}$ FXa and $500 \mathrm{nM}$ prothrombin. RNA was isolated from macrophages using TRIzol Reagent. Transcript levels were quantified by real time PCR.

\section{Statistical analyses}

Data are indicated as mean \pm standard error of the mean (SEM). Unpaired Student's t-test and two-way ANOVA were used as appropriate. For comparison of genotype distributions, a $\mathrm{X}^{2}$-test was applied. A $P$-value $<0.05$ was considered significant. 


\section{Results}

\section{Generation of C213G Tf KI mice}

To generate mice expressing C213G Tf under the control of the endogenous $\mathrm{Tf}$ promoter, a replacement-type targeting vector was cloned. This vector contained the coding sequence of C213G full length $T$ f, which was flanked by $3 \mathrm{~kb} 5^{\prime}$ and $5.2 \mathrm{~kb} \mathrm{3'}$ arms of homology; thus, the endogenous $T f$ sequence was replaced by the C213G TF coding sequence by homologous recombination (Figure 1A).
Partial developmental lethality in homozygous C213G Tf offspring

Heterozygous mice were bred, and among 889 offspring on a C57BL/6J genetic background, 141 homozygous C213G/C213G (16.1\%) Tf, 508 heterozygous C213G/wt Tf $(57.5 \%)$, and $240(26.4 \%)$ wt animals were found at weaning. At birth, among 320 offspring, 56 homozygous C213G/C213G (17.5\%), 170 heterozygous C213G/wt $(53.1 \%)$, and $94 \mathrm{wt}(29.4 \%)$ were found, excluding significant post-natal lethality (Figure 1B). Thus, one third of homozygous C213G/C213G pups were missing at birth

A

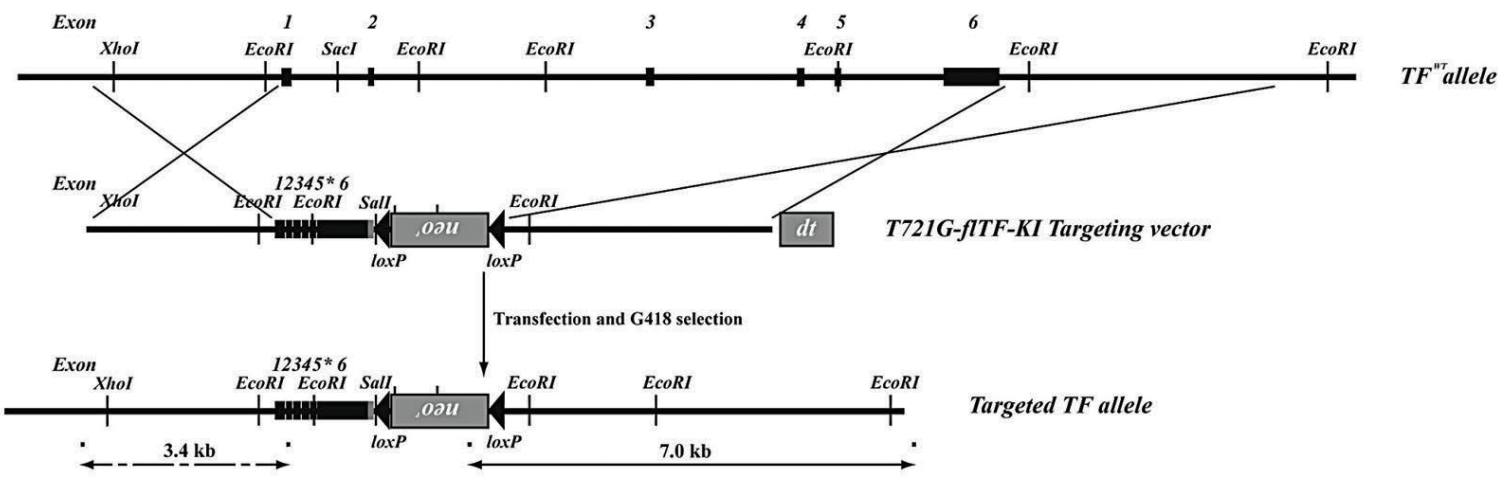

B

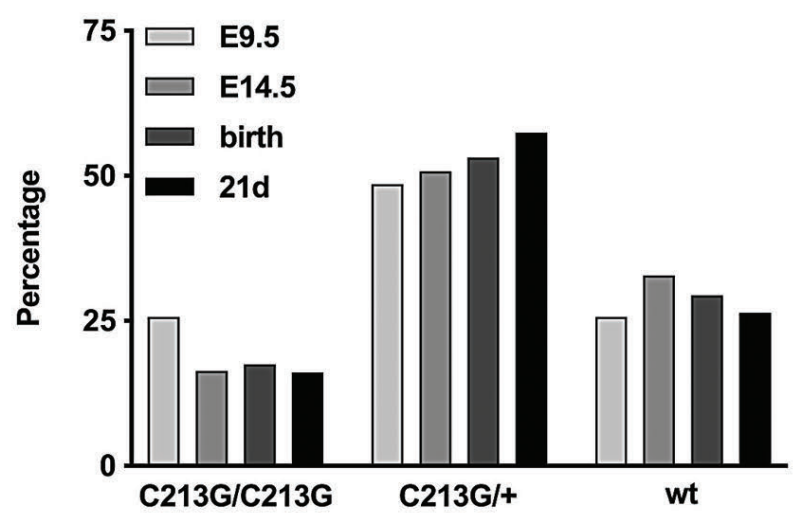

\begin{tabular}{|c|c|c|c|c|}
\hline & $\begin{array}{c}\text { C213G/ } \\
\text { C213G }\end{array}$ & C213G/+ & wt & n \\
\hline E9.5 & $25.7 \%$ & $48.6 \%$ & $25.7 \%$ & 70 \\
\hline E14.5 & $16.4 \%$ & $50.8 \%$ & $32.8 \%$ & 67 \\
\hline birth & $17.5 \%$ & $53.1 \%$ & $29.4 \%$ & 320 \\
\hline d21 & $16.1 \%$ & $57.5 \%$ & $26.4 \%$ & 889 \\
\hline & \multicolumn{4}{|c|}{} \\
\hline $\mathbf{X}^{2}$-test & E14.5 & birth & $\mathbf{2 1 d}$ & mendel \\
\hline E9.5 & 0.3642 & 0.2808 & 0.0963 & 0.9718 \\
\hline E14.5 & - & 0.8521 & 0.5379 & 0.163 \\
\hline birth & & - & 0.4607 & 0.0058 \\
\hline 21d & & & - & 0 \\
\hline
\end{tabular}

C

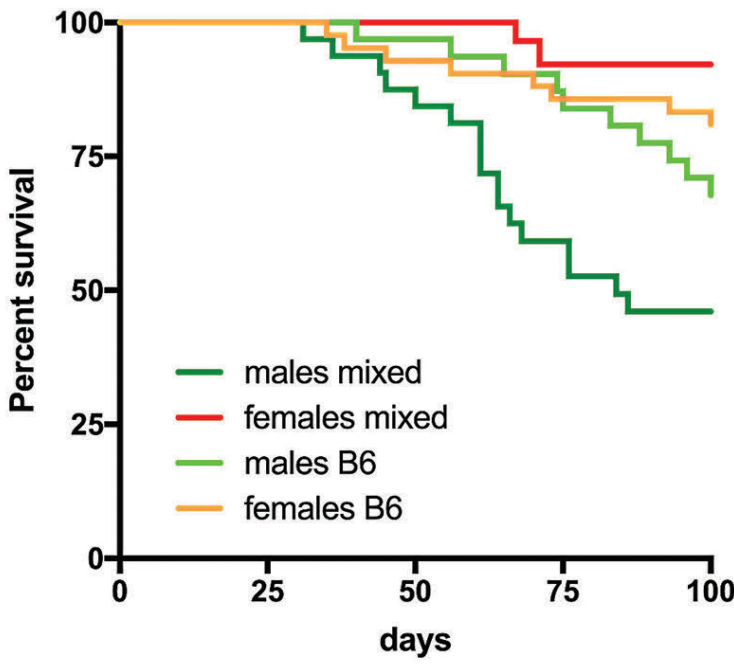

Figure 1. Genetic targeting, genotype distributions and survival. (A) The murine $\mathrm{Tf}$ allele was targeted with a replacement-type vector containing the murine fITF coding sequence with T721G nucleotide exchange in exon 5, flanked by $3 \mathrm{~kb} 5$ ' and $5.2 \mathrm{~kb} 3$ ' homology arms. Diphtheria toxin (dt) was used for negative selection. After homologous recombination into 129P2/OlaHsd embryonic stem cells, a loxP flanked neomycin resistance cassette was removed by transfection with a Cre expression plasmid. (B) Genotype distribution of offspring from heterozygous breeding pairs on a B6 background were analyzed at different time points. Genotype distributions were compared to a mendelian 25\%/50\%/25\% distribution as well as to the distributions at other time points. (C) Reduced survival of homozygous C213G Tf mice. Survival curve of homozygous male and female $\mathrm{C} 213 \mathrm{G} / \mathrm{C} 213 \mathrm{G}$ Tf offspring on mixed and B6 genetic background (male mixed $n=32$, female mixed $n=32$, male $B 6 n=32$, female B6 $n=43$ ). Dead and diseased mice meeting the termination criteria were both included in the analysis. 
A

Males

Females

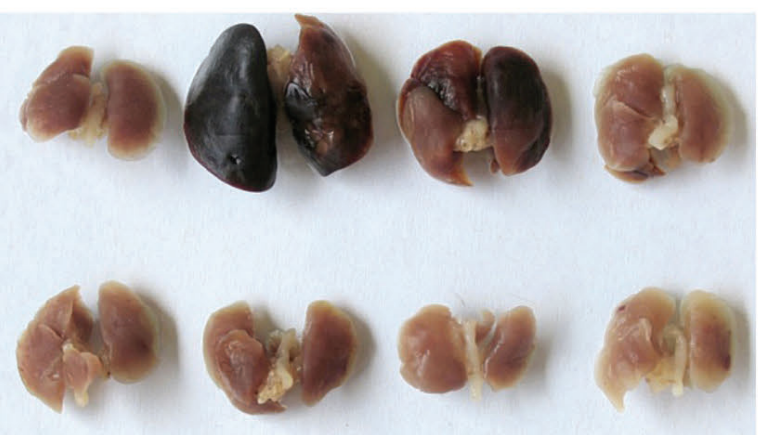

B

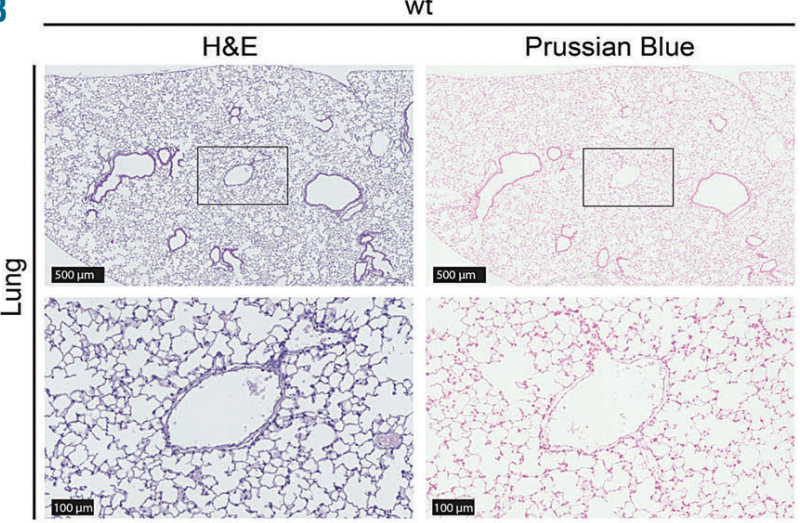

C

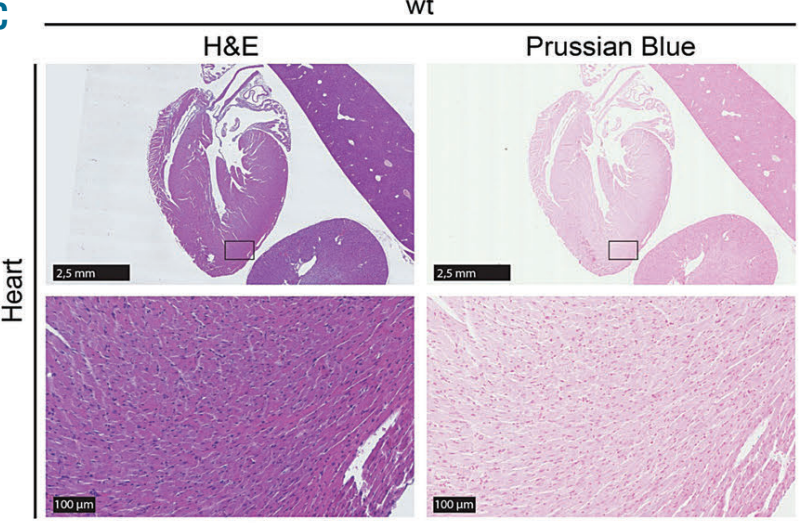

D

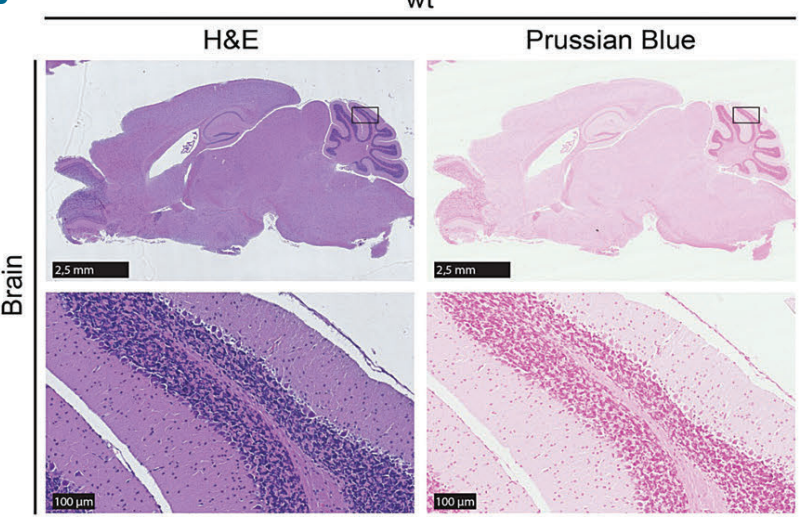

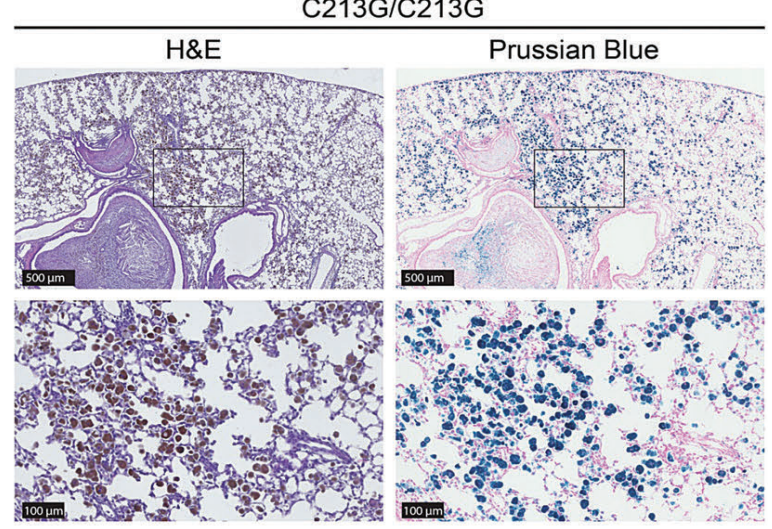

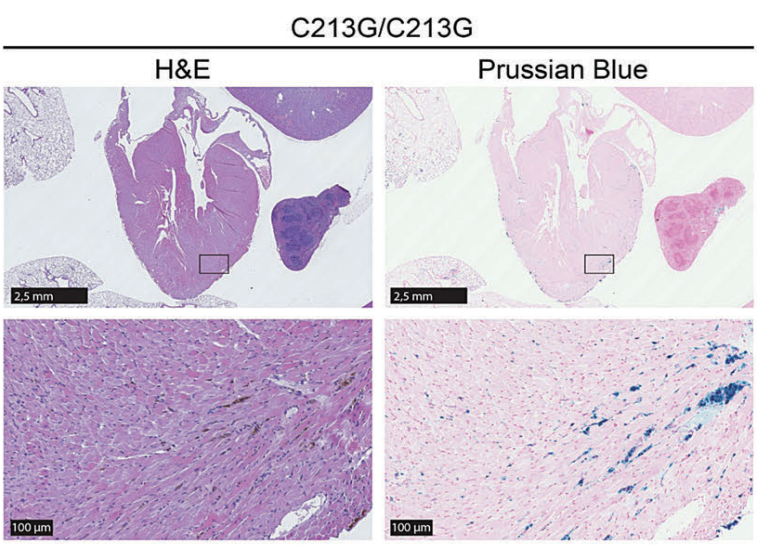

$\mathrm{C} 213 \mathrm{G} / \mathrm{C} 213 \mathrm{G}$

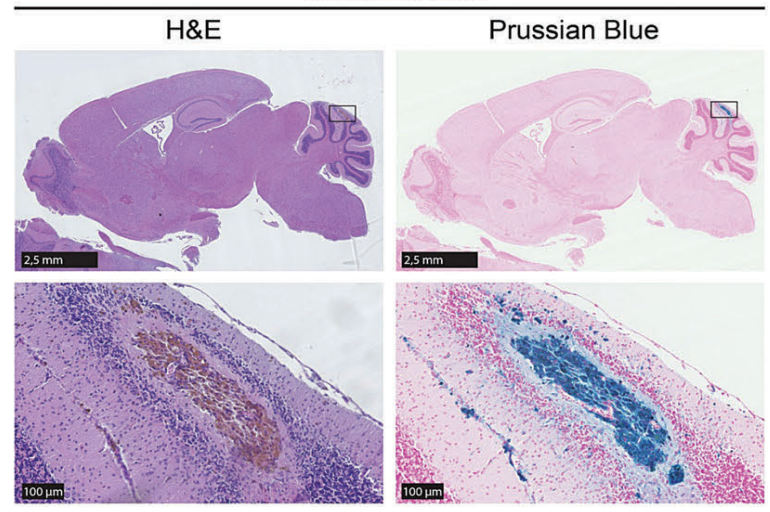

Figure 2. Macroscopic images of lung hemorrhages and iron detection (Prussian blue staining) in heart, lung and brain hemorrhages. (A) Representative macroscopic images of lung bleedings in male and female homozygous C213G/C213G Tf mice. (B) Lung, (C) heart, and (D) brain consecutive sections from wild-type (wt) and homozygous C213G/C213G Tf mice were stained with hematoxylin and eosin (H\&E) for morphological analysis and Prussian blue to show ferric iron $\left(\mathrm{Fe}^{+}\right)$in blood. The area of the higher magnification pictures $(20 \mathrm{x})$ is demarcated with a rectangle in the lower magnification ones (aprox. 1.45x in heart/brain and $5 x$ in lung). Iron deposits within lung macrophages are particularly obvious in (B). 
and both genotype distributions at weaning and at birth differed significantly from a mendelian distribution.

Genetic deletion of the Tf gene in mice leads to embryonic lethality between E9.5 and E10.5. ${ }^{3}$ To determine whether homozygous C213G/C213G Tf embryos are generated at mendelian ratios, 70 E9.5 embryos on a B6 genetic background were genotyped. Eighteen homozygous C213G/C213G Tf $(25.7 \%), 34$ heterozygous C213G/wt Tf (48.6\%), and $18 \mathrm{wt}(25.7 \%)$ embryos were found, in accordance with mendelian segregation (Figure $1 B)$. In contrast to $T f \mathrm{KO}$ mice, histology did not show any impairment of yolk sac vessel integrity and normal levels of -SMA expression in homozygous C213G/C213G Tf yolk sacs at E9.5 (Online Supplementary Figure S1A). ${ }^{3}$ At E14.5, 11 vital homozygous C213G/C213G TF (16.4\%), 34 heterozygous $\mathrm{C} 213 \mathrm{G} / \mathrm{wt}(50.8 \%)$ and $22 \mathrm{wt}$ embryos $(32.8 \%)$ were found, indicating that C213G/C213G Tf mice show partial developmental lethality between day E9.5 and E14.5. Embryonic growth required umbilical blood flow via the chorioallantoic placenta from day E10.5. ${ }^{31}$ Besides the 11 vital homozygous C213G/C213G Tf embryos, three necrotic homozygous embryos could be identified on day 14.5 showing retinal pigmentation which indicates development to at least E11.5. ${ }^{32}$ Additional resorbed implantation sites were present, which could not be genotyped. Placentas of both vital and necrotic homozygous C213G/C213G Tf embryos showed hemorrhages of variable number and size (Online Supplementary Figure S1A).

\section{Decreased survival of adult homozygous C213G/C213G Tf offspring}

Between the age of 3-4 weeks, about 10\% homozygous C213G/C213G Tf mice on a B6 background developed a hydrocephalus, while this was not observed in C213G/C213G Tf animals on a mixed background (data not shown). This may be due to the tendency of wt B6 mice to develop spontaneous hydrocephalous. ${ }^{33}$

With progressing age some homozygous C213G/C213G Tf mice developed symptoms of disease, mostly dyspnea, apathy and weight loss, but sometimes also neurological symptoms or spontaneous death. Diseased animals were euthanized when they met defined termination criteria. Both euthanized animals and those which died were included in the survival analysis. We found a sex difference in survival with impaired survival of male homozygous C213G/C213G Tf mice. The sex difference was more pronounced in animals on a mixed background $(P<0.001)$ compared to animals on a B6 background ( $P=0.17$, Figure $1 C)$. Dissection of dead and euthanized mice showed macroscopic bleedings to heart, lung, and brain, which are organs with high TF expression. Hemorrhages were confirmed microscopically in heart, lung, and brain (Figure 2A-D). They were accompanied by secondary changes like epi- and myocarditis and fibrosis in hearts as well as edema (Figure 3A-B), bronchiolitis obliterans, and pneumonia in lungs.

\section{Sex difference in organ pathology}

In order to quantify the incidence of organ bleedings, we analyzed a group of male and female homozygous C213G/C213G Tf mice on a B6 background without apparent disease symptoms. This analysis of asymptomatic mice provided a measure for the incidence of organ bleedings in a uniform group of mice of both sexes of the same age. At 17 weeks of age we found a similar incidence of hemorrhages in the heart (7 of 11 in females and 5 of 10 in males) and brain (2 of 11 in females and 2 of 10 in males) of male and female homozygous C213G/C213G Tf mice, while the incidence of lung hemorrhage was more pronounced in males (5 of 10) compared to females (1 of 11) (Online Supplementary Figure S1B). Lungs of male homozygous C213G/C213G Tf mice also exhibited macroscopically more hemorrhagic areas in comparison to female mice and these were accompanied by edema in severe cases (Figure 2A). Histologically, affected areas showed intra-alveolar hemorrhage and the presence of macrophages, eosinophilic fibrillar material in bronchial lumen, and peri-vascular and peri-bronchial inflammatory infiltrates (Figure 2B). In contrast to reported calcified testes in low-TF mice 9, we did not observe calcification of testes (Online Supplementary Figure S2A).

Epi- and myocardial hemorrhages (Figure 2C) as well as inflammation representing myocarditis (Figure 3A) were observed in the hearts of homozygous C213G/C213G Tf mice. Furthermore, fibrotic changes were detectable in all the hearts of homozygous C213G/C213G Tf mice, even without acute hemorrhage or inflammation (Figure 3B).

However, the blood pressure and heart rate of male and female C213G/C213G Tf mice were comparable to that of male and female wt mice (Online Supplementary Figure S2B).

\section{Molecular changes in the heart and lung of C213G/C213G Tf mice}

Using Illumina Bead array, we assessed gene expression profiles in hearts and lungs of male and female homozygous C213G/C213G Tf mice compared to the mean expression values of wt Tf mice (Figure $3 \mathrm{C}$ and Online Supplementary Table S1). 113 genes were significantly regulated in male and 178 genes in female C213G/C213G Tf mouse hearts. GO-terms which were both overrepresented in hearts of homozygous male and female C213G/C213G Tf mice were cellular movement, hematological system, immune cell trafficking, cancer, cardiovascular system, cellular growth and proliferation, skeletal and muscular disorders, cell-to-cell signaling and interaction, metabolic disease, cardiovascular disease, respiratory disease, and cell death. This is in line with inflammation and fibrotic remodeling. In male C213G/C213G Tf mouse lungs 69 genes were significantly upregulated and classified to the following overrepresented GO terms: inflammatory response, cellular movement, cell-to-cell signaling, hematological system, connective tissue, cancer, respiratory disease, cardiovascular disease, and lipid metabolism. In contrast, in female C213G/C213G Tf mouse lungs only 23 genes were weakly regulated (fold change of -3.662.10) while no GO term was overrepresented. Although the macroscopically least affected lungs were selected for the analysis, the differential gene regulation in male C213G/C213G Tf lungs compared to females suggests increased early pathological alterations and supports the sex difference in lung pathology.

\section{Tail bleeding time and blood cell counts}

Tail bleeding was measured to investigate whether primary differences exist in hemostatic capacity. Tail bleeding time was prolonged in homozygous C213G/C213G Tf mice of both sexes, but no difference was detected between the sexes (Figure 4A). Comparing blood counts 
A

wt
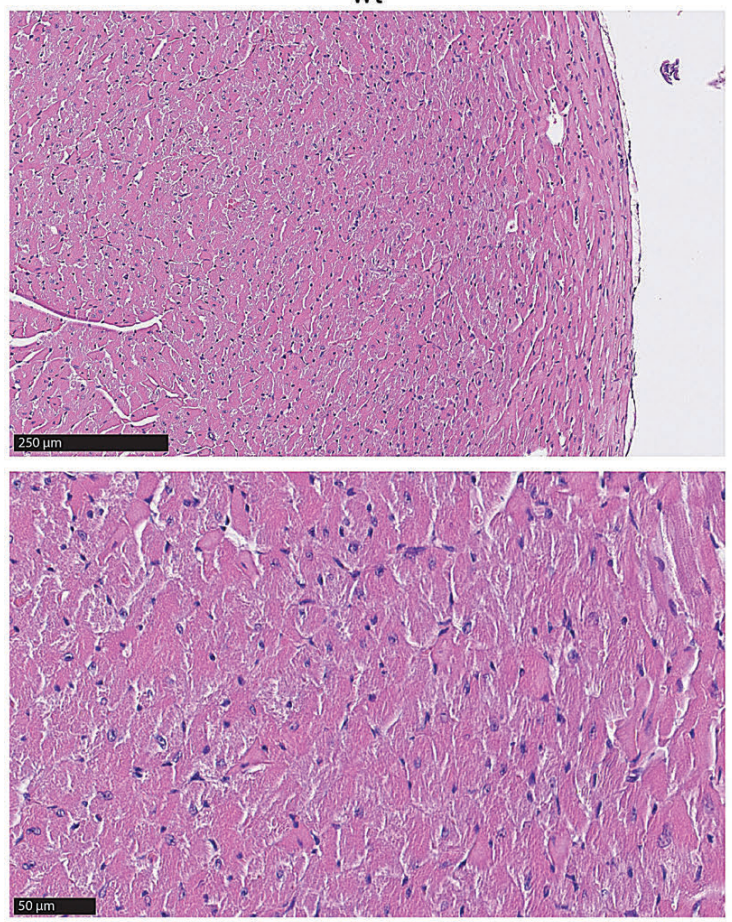

B

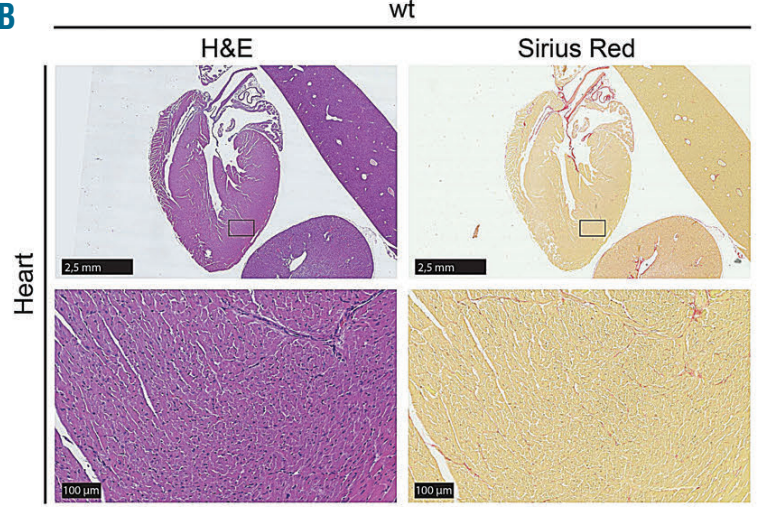

C213G/C213G

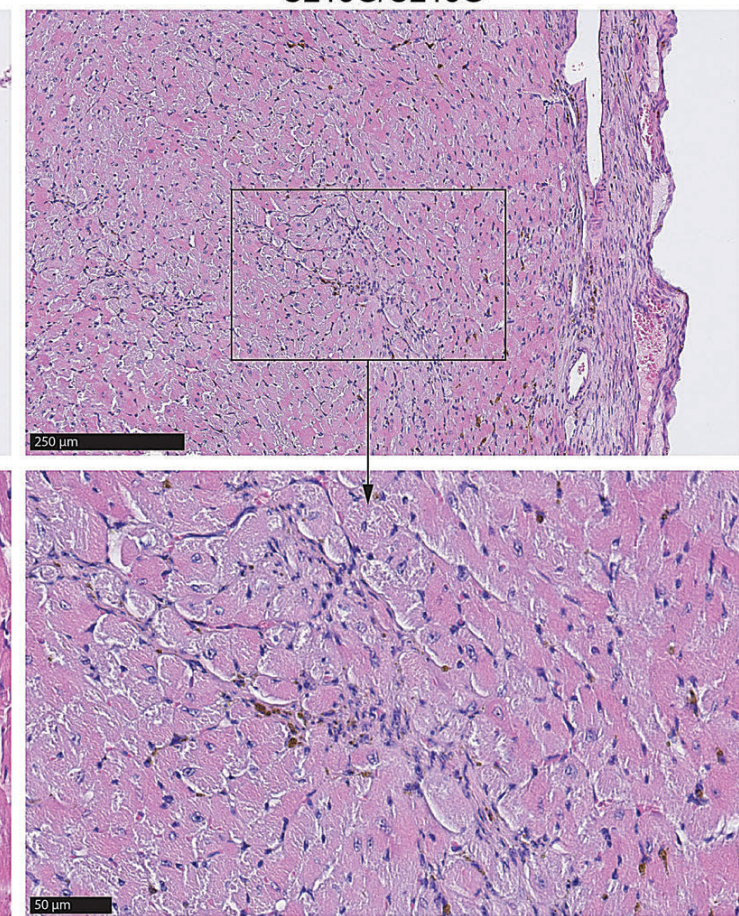

C213G/C213G

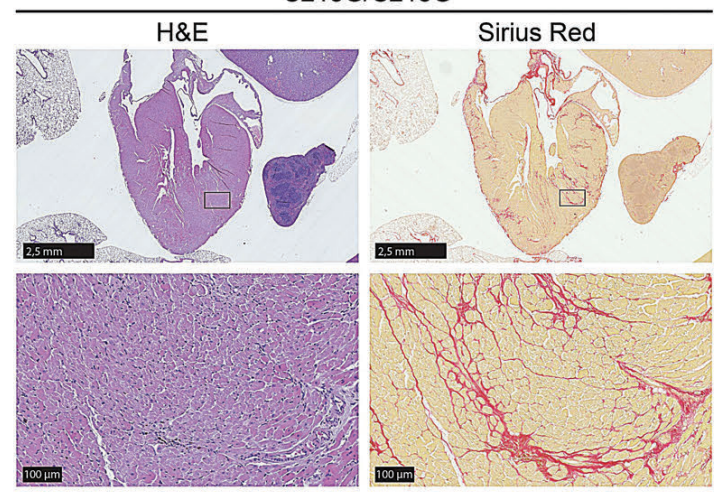

C

heart

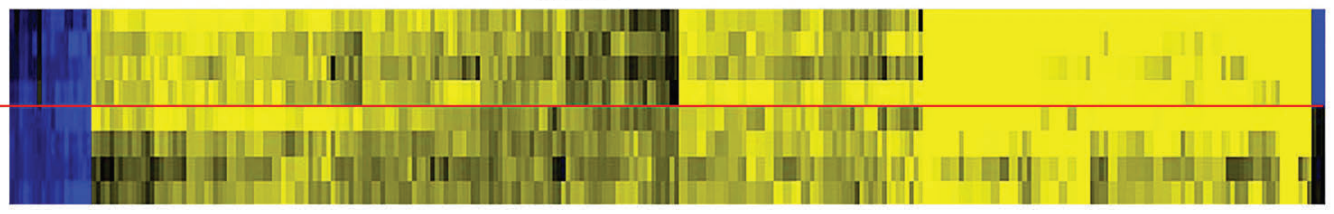

lung

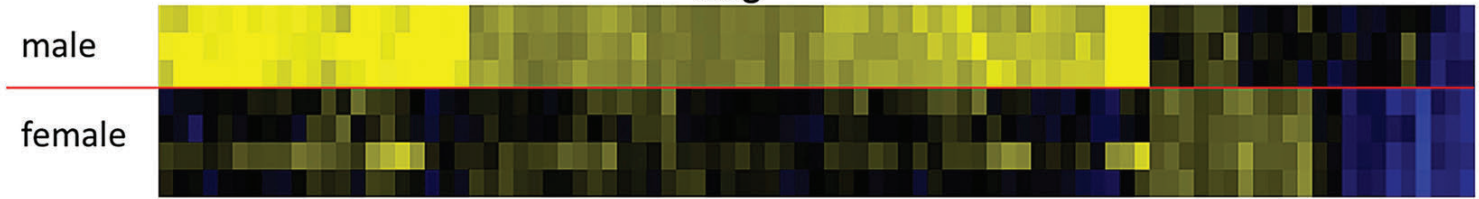

Figure 3. Histological images of myocarditis and myocardial fibrosis, gene profile in heart and lung tissue. (A) Heart sections of wild-type (wt) and homozygous C213G/C213G Tf mice were stained with hematoxylin and eosin (H\&E). Immune infiltrates are visible in the myocardium of C213G/C213G Tf animals. The area of the higher magnification picture (40x) is shown with a rectangle in the lower magnification one (15x). (B) Consecutive sections from wt and homozygous C213G/C213G TF mice were stained with H\&E for morphological analysis and Picro-sirius red for better visualization of collagen. The area of the higher magnification (20x) pictures is demarcated with a rectangle in the lower magnification ones (approx. 1.45x). (C) Heatmaps of significantly (FDR<10\%) regulated genes between homozygous C213G/C213G Tf and wt mice in heart and lung tissues. Genes are shown, if they are regulated in either male or female animals per tissue. Yellow (blue) indicates down (up) regulation compared to the mean expression of wt mice. 
of C213G/C213G Tf mice to wt mice revealed a mild decrease of cellular hemoglobin content $(\mathrm{MCH})$ and concentration (MCHC), slightly increased reticulocyte numbers with more immature reticulocytes, and an increased mean platelet size due to a higher proportion of big platelets (>12fl) in homozygous C213G/C213G Tf mice (Figure 4B). Even though these findings are consistent with compensated chronic bleedings, the absolute change of the values was small.

\section{Tf expression and activity}

Tf mRNA expression was measured in the heart, brain, and lung of male and female homozygous C213G/C213G
Tf and wt mice using flTF specific primers (Figure 5A). flTF expression was slightly elevated in the brain and lung of homozygous C213G/C213G Tf mice compared to wt mice. In the hearts of male C213G/C213G Tf mice, flTf mRNA was significantly increased 9.6-times compared to hearts of wt mice suggesting a compensatory transcriptional induction of a functionally insufficient protein that may involve recently demonstrated feedback loops of the TF cytoplasmic domain. ${ }^{34}$ In C213G/C213G Tf female hearts a similar induction was seen, however with a higher variability.

Induction of $T f$ transcription is in line with inflammatory and fibrotic remodeling seen in all hearts of both gen-
A

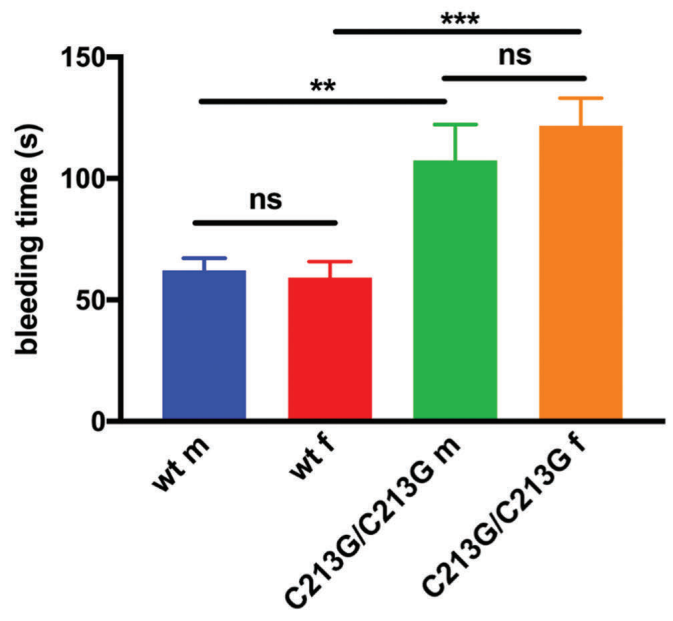

B

\begin{tabular}{|c|c|c|c|c|}
\hline \multirow{2}{*}{$\begin{array}{l}\text { Group: fITF (6 months) } \\
\text { additional group }\end{array}$} & \multicolumn{2}{|c|}{$\begin{array}{l}\text { Control } \\
\text { (A) }\end{array}$} & \multicolumn{2}{|c|}{$\begin{array}{l}\text { Mutant } \\
\text { (B) }\end{array}$} \\
\hline & Male & Female & Male & Female \\
\hline Number of animals tested & 11 & 10 & 8 & 7 \\
\hline WBC $\left[10^{3} / \mu L\right]$ & $9.2 \pm 0.62$ & $5.9 \pm 0.47$ & $9.1 \pm 0.49$ & $6.5 \pm 0.84$ \\
\hline 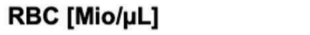 & $9.7 \pm 0.2$ & $10.1 \pm 0.11$ & $10.1 \pm 0.22$ & $9.9 \pm 0.17$ \\
\hline PLT $\left[10^{3} / \mu \mathrm{L}\right]$ & $1,942 \pm 70$ & $1,453 \pm 27.9$ & $1,860 \pm 111.4$ & $1,640 \pm 59.7$ \\
\hline Hemoglobin [g/dL] & $14 \pm 0.22$ & $14.7 \pm 0.15$ & $14.1 \pm 0.21$ & $14.1 \pm 0.22$ \\
\hline Hematocrit [\%] & $47 \pm 0.91$ & $48.8 \pm 0.5$ & $48.7 \pm 0.71$ & $47.5 \pm 0.65$ \\
\hline MCV [fL] & $48.3 \pm 0.35$ & $48.4 \pm 0.1$ & $48.3 \pm 0.57$ & $48 \pm 0.22$ \\
\hline MCH [pg] & $14.4 \pm 0.11$ & $14.6 \pm 0.06$ & $14 \pm 0.19$ & $14.3 \pm 0.11$ \\
\hline $\mathrm{MCHC}[\mathrm{g} / \mathrm{dL}]$ & $29.9 \pm 0.25$ & $30.1 \pm 0.14$ & $29 \pm 0.14$ & $29.7 \pm 0.17$ \\
\hline RDW-CV [\%] & $22.1 \pm 0.52$ & $21.6 \pm 0.19$ & $22.8 \pm 0.44$ & $21.7 \pm 0.21$ \\
\hline MPV [fL] & $6.58 \pm 0.03$ & $6.66 \pm 0.07$ & $6.79 \pm 0.08$ & $6.77 \pm 0.09$ \\
\hline PDW [fL] & $7.11 \pm 0.05$ & $7.2 \pm 0.09$ & $7.39 \pm 0.11$ & $7.23 \pm 0.12$ \\
\hline P-LCR [\%] (PV>12fL) & $4.28 \pm 0.15$ & $4.81 \pm 0.34$ & $5.31 \pm 0.37$ & $5.21 \pm 0.45$ \\
\hline $\operatorname{Ret}[\mathrm{Mio} / \mu \mathrm{L}]$ & $0.43 \pm 0.02$ & $0.44 \pm 0.03$ & $0.48 \pm 0.03$ & $0.56 \pm 0.06$ \\
\hline Ret [\%] & $4.38 \pm 0.17$ & $4.4 \pm 0.22$ & $4.75 \pm 0.31$ & $5.69 \pm 0.64$ \\
\hline LF Ret [\%] & $42.65 \pm 0.96$ & $43.63 \pm 0.62$ & $41.4 \pm 0.99$ & $41.27 \pm 0.83$ \\
\hline MF Ret [\%] & $17.29 \pm 0.42$ & $18.38 \pm 0.51$ & $18.66 \pm 0.23$ & $19.14 \pm 0.52$ \\
\hline HF Ret [\%] & $40.06 \pm 1.04$ & $37.99 \pm 0.85$ & $39.94 \pm 1$ & $39.59 \pm 0.79$ \\
\hline Immature Ret [\%] & $57.35 \pm 0.96$ & $56.37 \pm 0.62$ & $58.6 \pm 0.99$ & $58.73 \pm 0.83$ \\
\hline
\end{tabular}

Figure 4. Bleeding time and blood counts (A) Bleeding times after amputation of 5 $\mathrm{mm}$ tail tip; male wt $\mathrm{n}=19$, female wt $\mathrm{n}=13$, male C213G/C213G $n=15$, female $\mathrm{C} 213 \mathrm{G} / \mathrm{C} 213 \mathrm{G} \quad \mathrm{n}=11 ; \quad * * P<0.01$ $* * * P<0.001$. (B) Blood count analysis in C213G/C213G Tf and wt mice in with Sysmex XT-2000iV. Some of the values where significantly different between C213G/C213G Tf mice and wt Tf mice. However, absolute differences were all minor. 
ders. To assess the protein expression and activity of C213G TF, organ lysates were prepared from heart, brain and lung. Protein expression of C213G TF was 10-times lower than expression of wt TF in brain and lung. In hearts, protein expression of C213G TF and wt TF did not differ (Figure 5B), in line with the compensatory upregulation of gene transcription. Pro-coagulant activity was measured using a plasma clotting assay. Pro-coagulant activity of brain and lung lysates from C213G/C213G Tf mice was decreased about 1000-fold compared to wt Tf brains and lungs. In heart lysates, the difference in procoagulant activity was only about 100 -fold (Figure 5C). A 10 -fold reduction of protein expression in brains and lungs of C213G/C213G Tf mice is in line with a 10-fold stronger decrease in pro-coagulant activity compared to the heart. In both genotypes, there was no difference between the sexes, neither in expression nor in pro-coagulant activity.

\section{The TF allosteric disulfide is required for $T f$} procoagulant function in macrophages

We characterized the pro-coagulant activity of the C213G Tf mutant in primary bone marrow-derived macrophages. In order to assure unaltered induction of $T f$ in IFN primed and 4 hour (h) lipopolysaccharides (LPS) stimulated macrophages, ${ }^{29,30}$ we measured Tf mRNA levels with flTF-specific primers (Figure 6A). flTf mRNA levels were significantly higher in C213G/C213G TF macrophages which is expected as in these cells both alleles of endogenous Tf (yielding fully processed mRNA for fITF and as TF) were replaced with a gene that only yields the mutated flTf. Given the unimpaired mRNA induction, we next measured TF protein expression 90 minutes (min) and $4 \mathrm{~h}$ after LPS stimulation (Figure $6 \mathrm{~B}$ ). While TF induction was below the detection limit in the absence of IFN $\gamma$ priming, a low molecular $\sim 48 \mathrm{kDa}$ form of TF was expressed after 90 min of stimulation in both IFN $\gamma$-primed wt and C213G/C213G Tf macrophages. After $4 \mathrm{~h}$ of stimulation, wt TF was predominantly expressed as a highly glycosylated protein which was not detected in macrophages from C213G/C213G Tf mice.

Since glycosylation of TF is required for cell surface expression and procoagulant activity in intestinal epithelial cells, ${ }^{35}$ we next tested the procoagulant activity of intact macrophages. Macrophage cell surface TF is largely inactive and requires activation of the $\mathrm{P} 2 \mathrm{X} 7$ receptor with ATP. C213G/C213G Tf macrophages displayed minimal procoagulant activity on their cell surface after ATP stimulation and no TF activity or antigen was detected on EV released from these cells (Figure 6C-D). Western blotting for other proteins typically released on ATP-induced EV $^{36}$ revealed no quantitative abnormalities in EV release from C213G/C213G Tf macrophages, demonstrating that TF is selectively absent in EV derived from C213G/C213G Tf macrophages (Figure 6D). In addition, measurements of prothrombinase activity showed that the release of procoagulant lipids on EV was not different between wt and C213G/C213G Tf macrophages (Figure 6C). Thus, mutation of the allosteric disulfide of TF prevents glycosylation and normal cellular processing, leading to a severely impaired procoagulant activity in macrophages stimulated with inflammatory mediators.

\section{Behavioral differences}

Having excluded that primary differences in protein expression and activity as well as general hemostatic
A

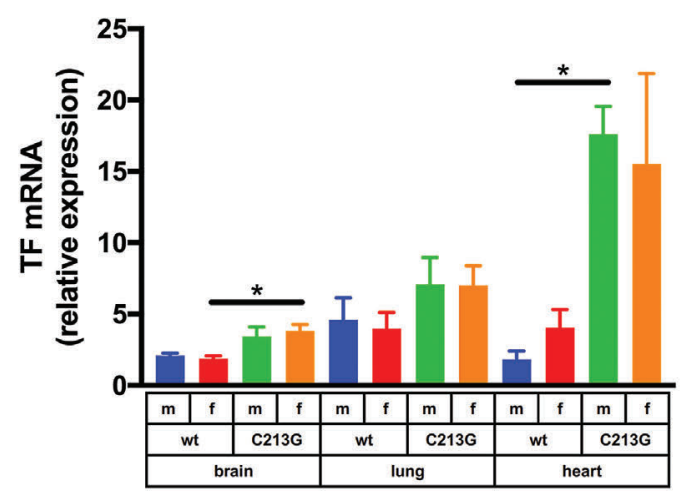

B
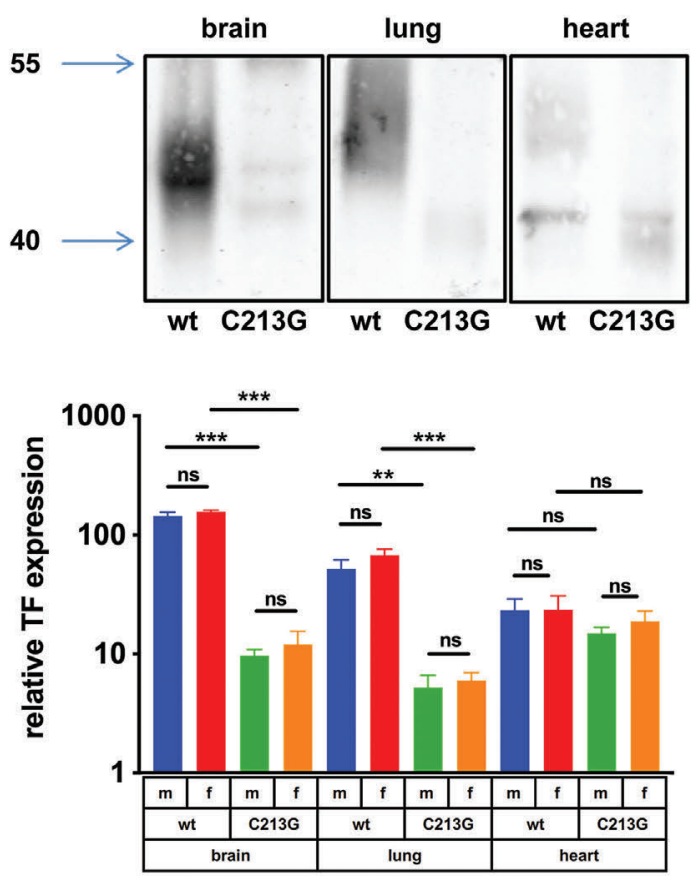

C

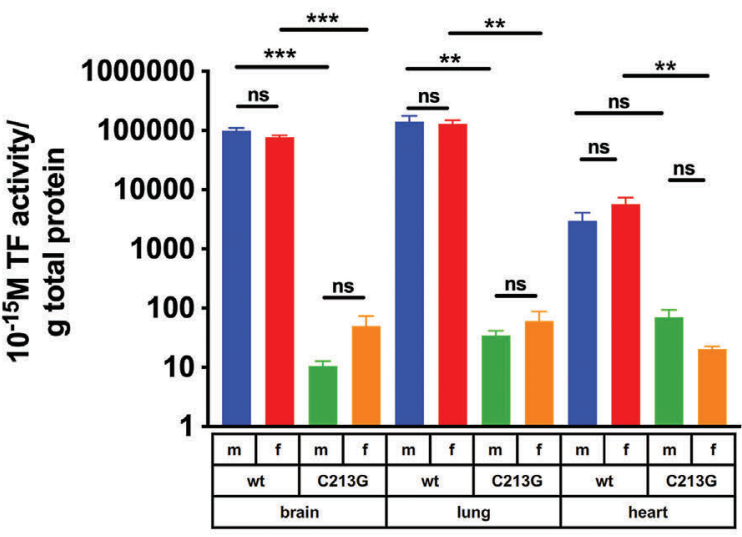

Figure 5. TF expression and activity in brain, lung and heart. (A) Semi-quantitative RT-PCR in brain, lung, and heart of male and female homozygous C213G/C213G TF mice and wt TF mice using fITF-specific primers and normalization for $\beta$-actin $(n=6, * P<0.05)$. (B) Representative blots and quantification of TF protein expression in brain, lung and heart of male and female homozygous C213G/C213G TF mice and wt TF mice $(n=4, * * P<0.01, * * * P<0.001)$. (C) Plasma clotting time measurements of TF activity in brain, lung and heart of male and female homozygous $\mathrm{C} 213 \mathrm{G} / \mathrm{C} 213 \mathrm{G}$ TF and wt TF mice $(\mathrm{n}=4$ $* * P<0.01, * * * P<0.001)$. 
A

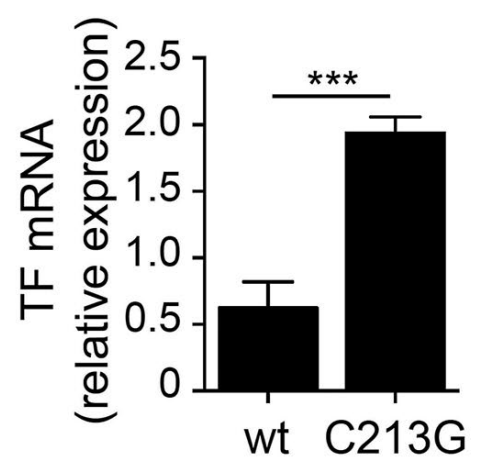

B

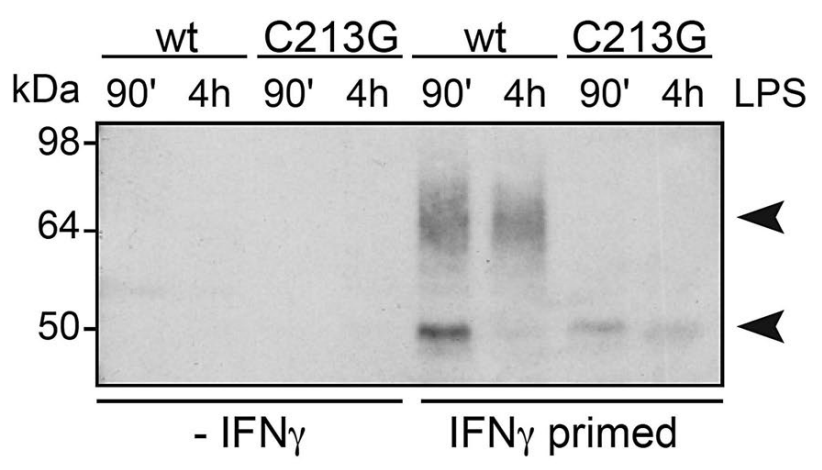

C

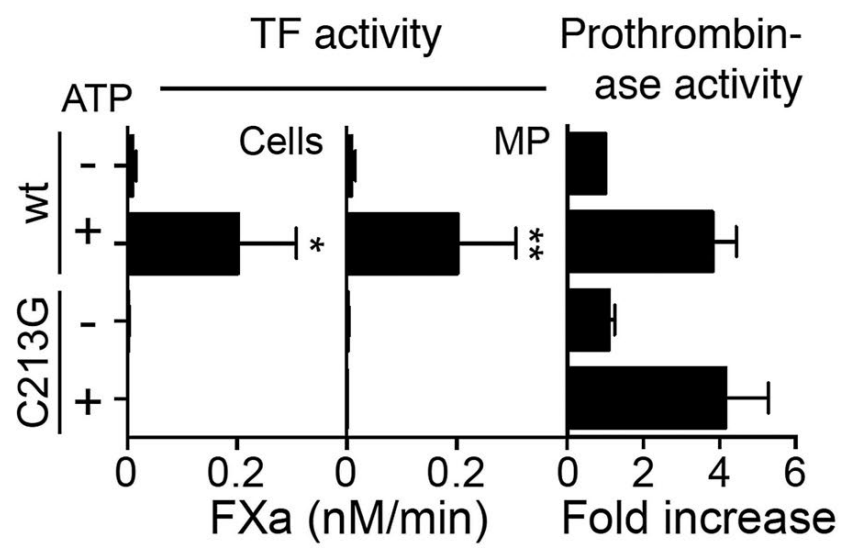

D

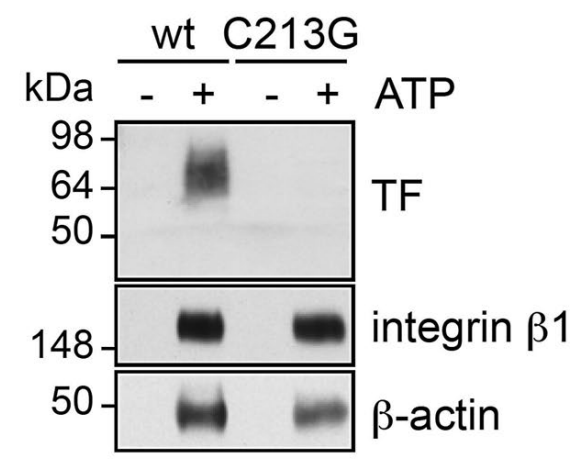

Figure 6. TF expression and extracellular vesicle release in bone marrow-derived macrophages. (A) TF mRNA levels were determined by semi-quantitative RT-PCR using fITF-specific primers and normalization for $\beta$-actin. Bone marrow-derived macrophages cultured overnight without or with IFN $\gamma$ (100 ng/mL) were stimulated with $1 \mathrm{mg} / \mathrm{mL}$ LPS for 4 hours (h); wild-type (wt) $n=5, C 213 \mathrm{G} / \mathrm{C} 213 \mathrm{G} n=4 ; * * * P<0.001$. (B) Western-blotting for TF with a polyclonal anti-mouse TF antibody of membrane fractions from macrophages with or without overnight IFN $\gamma$ priming stimulated with $1 \mathrm{mg} / \mathrm{mL}$ lipopolysaccharides (LPS) for the indicated times (representing two independent experiments). (C) TF cellular and extracellular vesicle (EV) activity measured by FXa generation assay of INF $\gamma$ primed and LPS stimulated macrophages with or without activation of the P2X7 receptor with $5 \mathrm{mM}$ ATP for 30 minutes (min); $n=3, * P<0.05, * * P<0.01$. Prothrombinase activity of the same EV preparations was determined as a measure for the release of procoagulant, phosphatidylserine (PS)-expressing MP. (D) Protein composition of EV released after ATP stimulation for 30 min was evaluated by Western blotting for TF, integrin $\beta 1$, and $\beta$-actin. Densitometric quantification of protein levels from three independent experiments showed that levels on EV from C213G/C213G TF macrophages were 1.4 \pm 1.1 for integrin $\beta 1$ and $1.4 \pm 1.7$ for $\beta$-actin relative to wt-derived EV levels (representing two independent experiments).

capacity exist between the sexes, we investigated whether behavioral differences of male and female mice may underlie differences in disease severity in homozygous C213G/C213G Tf mice. At the age of 21 days, 12 homozygous C213G/C213G Tf males were weaned to individual cages and housed individually up to the age of 100 days to prevent aggressive fighting. All 12 males survived to the age of 100 days, which is a significant improvement compared to group-housed males $(P=0.0326)$ (Figure 7A). After day 100, pulmonary hemorrhages were detected in 3 of $12(25 \%)$ single-housed homozygous C213G/C213G Tf males (Figure 7B), in comparison to $50 \%$ lung hemorrhages in homozygous C213G/C213G Tf group housed males. This experiment demonstrates that lung hemorrhages develop spontaneously in homozygous C213G/C213G Tf males, but single housing reduces the risk for lung bleedings and lowers mortality.

\section{Discussion}

After the description of the importance of the TF C-ter- minal disulfide bridge for TF pro-coagulant activity ${ }^{26}$ and evidence for its involvement in TF decryption ${ }^{20,21,27}$ it is crucial to understand its significance in an in vivo model. In the C213G Tf mouse model we observed changes similar to those previously reported in vitro: reduced protein expression, glycosylation, and surface translocation. In line with this, the pro-coagulant activity is reduced by at least a factor 100 independent of changes in protein expression. The C213G Tf mutant still exhibits sufficient pro-coagulant activity to ensure embryonic development in two thirds of the offspring and to allow early post-natal survival.

The overall phenotype of C213G/C213G Tf mice shows impressive similarity to the phenotype of low-Tf mice, which express a human TF transgene in the absence of mouse Tf. Pro-coagulant activity in low-TF mice was estimated $1 \%$ of normal levels resulting from low expression of TF under the control of the human promoter. ${ }^{6}$ However, low-TF mice are born in accordance with Mendelian ratios, ${ }^{6}$ while C213G Tf mice displayed a reduced number of homozygous C213G/C213G Tf offspring at birth. Partial developmental lethality is observed when other clotting factors (FV, prothrombin) or the 
A

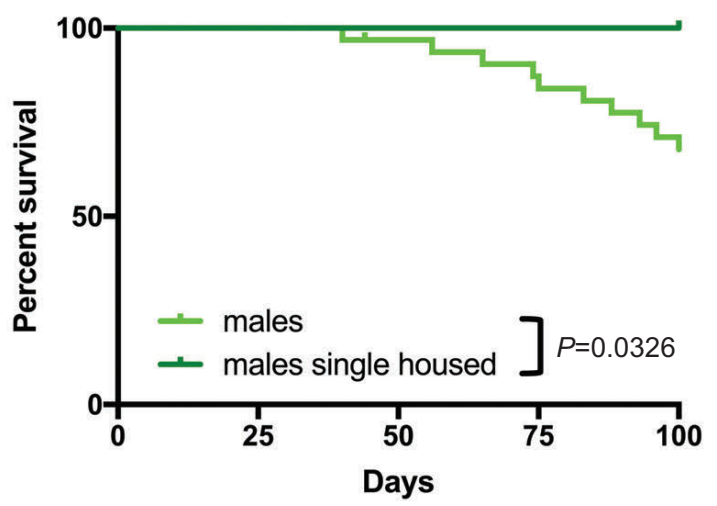

B

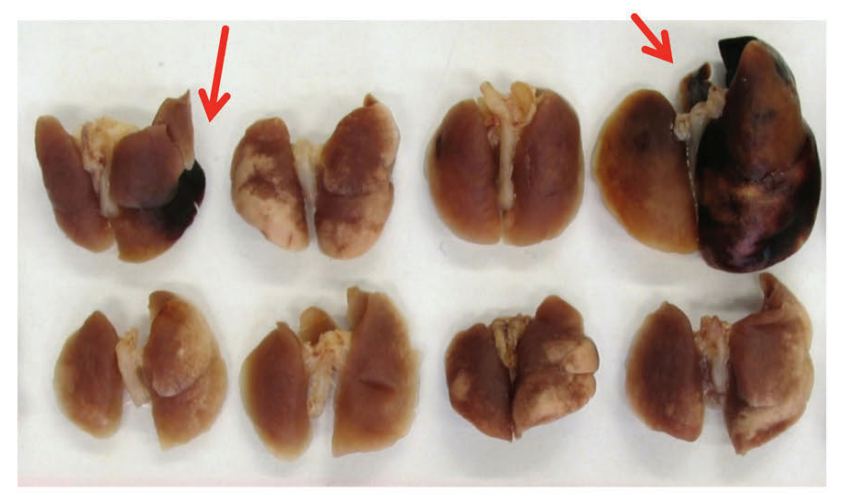

Figure 7. Single housing reduces incidence of lung bleedings and lethality in homozygous C213G/C213G male mice. (A) All 12 single-housed C213G/C213G Tf males survived up to 100 days, in contrast to $\mathrm{C} 213 \mathrm{G} / \mathrm{C} 213 \mathrm{G}$ Tf males, which were group-housed $(\mathrm{n}=32)$. (B) Incidence of lung bleedings was reduced from $50 \%$ to $25 \%$ in homozygous C213G/C213G TF males.

thrombin receptor PAR1 are knocked out. ${ }^{37-39}$ Deficiency in PAR2, which is activated by the TF/FVIIa complex, results in a partial, but undefined developmental lethality, ${ }^{40}$ suggesting that thrombin generation and PAR1 signaling, but not PAR2 signaling, are crucial for development. Rescue experiments of murine TF (murine $T f$ ) $\mathrm{KO}$ using different human $T F$ transgenic lines suggested that the threshold of TF activity needed to support embryonic development is lower than that needed to maintain postpartum hemostasis, as some transgenic lines quantitatively rescue genetic deletion of murine $T f$ but rescued pups die days or weeks after birth. ${ }^{41}$ For C213G/C213G Tf embryos this either suggests that expression under the endogenous mTF promoter may lead to different spatiotemporal expression patterns associated with different thresholds or that certain signaling functions of C213G Tf are altered, whose importance for development has not been recognized yet. Given the placental hemorrhages observed in homozygous C213G/C213G Tf mice, placental insufficiency might contribute to the partial developmental lethality in the latter and is in line with the low-TF phenotype exhibiting blood pooling in placentas of lowTF embryos at E14.5. ${ }^{13}$

Reduced survival of low-TF mice was mainly attributed to impaired left ventricular function induced by myocardial hemorrhages and fibrosis. ${ }^{6}$ Development of cardiac fibrosis was found to be sex-dependent with lower severity in female mice and associated with downregulation of fibrinolytic urokinase plasminogen activator. ${ }^{42}$ Although there was a sex-dependent effect on the development of cardiac fibrosis, no difference in survival was described between the sexes. No changes in heart rate, systolic, diastolic, or mean arterial blood pressure was found in C213G/C213G Tf mice, suggesting that the myocardial fibrosis did not fully translate into cardiac dysfunction. At the mRNA level, cardiac Tf expression was about 10-times higher in C213G/C213G Tf mice compared to wt TF mice. This increase translated in a similar cardiac protein expression in C213G/C213G Tf mice and wt Tf mice - in contrast to lung and brain, where protein expression was lower in C213G/C213G Tf mice. This observation is consistent with a compensatory elevation of transcription and may in part explain the unaltered hemodynamic parameters.

C213G/C213G Tf males showed lower survival compared to females; however, from autopsies of dead or terminally ill mice it was difficult to define the exact cause of death, particularly when all the three organs brain, heart, and lung showed hemorrhages. We therefore analyzed the incidence of sub-lethal hemorrhages in a uniform cohort of male and female C213G/C213G Tf and wt Tf mice, assuming that the incidence of sub-lethal hemorrhages correlates with the incidence of terminal hemorrhages. In this healthy appearing cohort, we found a considerable degree of cardiac fibrosis in 100\% of C213G/C213G Tf mice, and the level of fibrosis did not differ between male and female mice. However, male C213G/C213G Tf mice showed a higher incidence of lung bleedings compared to females as well as a differential pulmonary gene expression pattern. This suggests that in C213G/C213G Tf mice, lung hemorrhages may represent the main reason for terminal disease. To address the question why male C213G/C213G Tf mice are more prone to lung bleedings than female C213G/C213G Tf mice, we investigated possible molecular differences in $T f$ expression between the sexes or functional differences in bleeding time. As neither of these parameters showed a difference between female and male homozygous C213G/C213G Tf mice, we hypothesized that behavioral differences, in particular aggressive fighting, leads to aggravated pulmonary hemorrhage in homozygous C213G/C213G Tf males. Inter-male aggression is a well known problem in housing of male laboratory mice, which can be modulated by genetic background as well as cage enrichment. ${ }^{43}$ Cases of exercise induced pulmonary hemorrhage exist in healthy humans ${ }^{44,45}$ and spontaneous pulmonary hemorrhages are a known complication of anticoagulant therapy. ${ }^{46-49}$ Single housing indeed reduced the incidence of pulmonary hemorrhages and significantly improved survival of homozygous C213G/C213G Tf male mice with low pulmonary TF activity, suggesting that fully active TF is protective against spontaneous and exercise induced pulmonary hemorrhages. A previous report suggested that fatal lung hemorrhages may also represent a major reason for 
reduced survival of low-TF mice. ${ }^{8}$ In line with this, $17 \%$ of low-TF mice die of spontaneous hemorrhages in lung, brain, and gastrointestinal tract., ${ }^{7,10}$

In contrast to low-TF mice, ${ }^{9}$ we did not observe any testis calcification in C213G/C213G Tf male mice. The morphology of the seminiferous tubules of the testis and the general sperm amount in the cauda epididymis seemed normal. Since the corresponding single Cys replacement in human TF is compatible with TF-FVIIa signaling, ${ }^{21}$ one possible explanation for these differences is preservation of integrin-dependent TF signaling ${ }^{50}$ in the context of testes physiology.

In conclusion, mutation of the allosteric disulfide bond of TF leads to some reduction in protein expression and a pronounced reduction in protein activity of at least 100 -fold which goes along with impaired glycosylation and cell surface expression. This impairment results in a bleeding phenotype causing partial developmental lethality and high susceptibility of the lung, brain, and heart to bleedings after birth. Male mice exhibit more frequent and severe lung hemorrhages, which can be reduced by single housing preventing inter-male aggression. Thus, this study emphasizes the importance of the allosteric disulfide for proper TF expression, modification and function in vivo and the importance of functional TF for developmental and post-natal hemostasis and survival.

\section{Funding}

GMC was supported by the German Federal Ministry of Education and Research (Infrafrontier grant 01KX1012 to MHA). WR is supported by the National Heart Lung Blood Institute (HL-60472). SHMS and FCT were supported by the Swiss Heart Foundation.

\section{References}

1. Drake TA, Morrissey JH, Edgington TS. Selective cellular expression of tissue factor in human tissues. Implications for disorders of hemostasis and thrombosis. Am J Pathol. 1989;134(5):1087-1097.

2. Mackman N, Sawdey MS, Keeton MR, Loskutoff DJ. Murine tissue factor gene expression in vivo. Tissue and cell specificity and regulation by lipopolysaccharide. Am J Pathol. 1993;143(1):76-84.

3. Carmeliet P, Mackman N, Moons L, et al. Role of tissue factor in embryonic blood vessel development. Nature. 1996; 383(6595): 73-75.

4. Bugge TH, Xiao Q, Kombrinck KW, et al. Fatal embryonic bleeding events in mice lacking tissue factor, the cell-associated initiator of blood coagulation. Proc Natl Acad Sci U S A. 1996;93(13):6258-6263.

5. Toomey JR, Kratzer KE, Lasky NM, Stanton JJ, Broze GJ, Jr. Targeted disruption of the murine tissue factor gene results in embryonic lethality. Blood. 1996;88(5):1583-1587.

6. Parry GC, Erlich JH, Carmeliet P, Luther T, Mackman N. Low levels of tissue factor are compatible with development and hemostasis in mice. J Clin Invest. 1998;101(3):560569.

7. Pawlinski R, Fernandes A, Kehrle B, et al. Tissue factor deficiency causes cardiac fibrosis and left ventricular dysfunction. Proc Natl Acad Sci U S A. 2002;99(24):15333-15338.

8. Pedersen B, Holscher T, Sato Y, Pawlinski R, Mackman N. A balance between tissue factor and tissue factor pathway inhibitor is required for embryonic development and hemostasis in adult mice. Blood. 2005; 105(7):2777-2782.

9. Mackman N. Tissue-specific hemostasis in mice. Arterioscler Thromb Vasc Biol. 2005; 25(11):2273-2281.

10. Bode MF, Mackman N. A combined deficiency of tissue factor and PAR-4 is associated with fatal pulmonary hemorrhage in mice. Thromb Res. 2016;146:46-50

11. Bastarache JA, Sebag SC, Clune JK, et al. Low levels of tissue factor lead to alveolar haemorrhage, potentiating murine acute lung injury and oxidative stress. Thorax. 2012;67(12):1032-1039

12. Antoniak S, Tatsumi K, Hisada Y, et al. Tissue factor deficiency increases alveolar hemorrhage and death in influenza A virusinfected mice. J Thromb Haemost. 2016; 14(6):1238-1248.

13. Erlich J, Parry GC, Fearns C, et al. Tissue factor is required for uterine hemostasis and maintenance of the placental labyrinth during gestation. Proc Natl Acad Sci U S A 1999:96(14):8138-8143

14. Pawlinski R, Pedersen B, Erlich J, Mackman $\mathrm{N}$. Role of tissue factor in haemostasis, thrombosis, angiogenesis and inflammation: lessons from low tissue factor mice. Thromb Haemost. 2004;92(3):444-450.

15. Osterud B. Tissue factor expression in blood cells. Thromb Res. 2010;125 Suppl 1:S31-34.

16. Swystun LL, Liaw PC. The role of leukocytes in thrombosis. Blood. 2016;128(6):753-762.

17. Bach RR. Tissue factor encryption. Arterioscler Thromb Vasc Biol. 2006; 26(3):456-461.

18. Pendurthi UR, Ghosh S, Mandal SK, Rao LV. Tissue factor activation: is disulfide bond switching a regulatory mechanism? Blood. 2007;110(12):3900-3908.

19. Kothari H, Nayak RC, Rao LV, Pendurthi UR. Cystine 186-cystine 209 disulfide bond is not essential for the procoagulant activity of tissue factor or for its de-encryption. Blood. 2010;115(21):4273-4283.

20. Chen VM, Ahamed J, Versteeg HH, Berndt MC, Ruf W, Hogg PJ. Evidence for activation of tissue factor by an allosteric disulfide bond. Biochemistry. 2006;45(39):12020 12028.

21. Ahamed J, Versteeg $\mathrm{HH}$, Kerver $\mathrm{M}$, et al Disulfide isomerization switches tissue factor from coagulation to cell signaling. Proc Natl Acad Sci U S A. 2006;103(38):13932 13937.

22. Reinhardt C, von Bruhl ML, Manukyan D, et al. Protein disulfide isomerase acts as an injury response signal that enhances fibrin generation via tissue factor activation. J Clin Invest. 2008;118(3):1110-1122.

23. Langer F, Spath B, Fischer C, et al. Rapid activation of monocyte tissue factor by antithymocyte globulin is dependent on complement and protein disulfide isomerase. Blood. 2013;121(12):2324-2335.

24. Subramaniam S, Jurk K, Hobohm L, et al. Distinct contributions of complement factors to platelet activation and fibrin formation in venous thrombus development. Blood. 2017;129(16):2291-2302

25. Muller-Calleja N, Ritter S, Hollerbach A,
Falter T, Lackner KJ, Ruf W. Complement C5 but not C3 is expendable for tissue factor activation by cofactor-independent antiphospholipid antibodies. Blood Adv. 2018;2(9):979-986.

26. Rehemtulla A, Ruf W, Edgington TS. The integrity of the cysteine 186-cysteine 209 bond of the second disulfide loop of tissue factor is required for binding of factor VII. J Biol Chem. 1991;266(16):10294-10299.

27. van den Hengel LG, Osanto S, Reitsma PH Versteeg HH. Murine tissue factor coagulant activity is critically dependent on the presence of an intact allosteric disulfide. Haematologica. 2013;98(1):153-158.

28. van den Hengel LG, van den Berg YW, Reitsma $\mathrm{PH}$, Bos $\mathrm{MH}$, Versteeg $\mathrm{HH}$. Evolutionary conservation of the tissue factor disulfide bonds and identification of a possible oxidoreductase binding motif. J Thromb Haemost. 2012;10(1):161-162.

29. Furlan-Freguia C, Marchese P, Gruber A, Ruggeri ZM, Ruf W. P2X7 receptor signaling contributes to tissue factor-dependent thrombosis in mice. J Clin Invest. 2011;121(7):2932-2944.

30. Rothmeier AS, Marchese P, Petrich BG, et al Caspase-1-mediated pathway promotes generation of thromboinflammatory microparticles. J Clin Invest. 2015;125(4): 1471-1484.

31. Mu J, Adamson SL. Developmental changes in hemodynamics of uterine artery, uteroand umbilicoplacental, and vitelline circulations in mouse throughout gestation. Am J Physiol Heart Circ Physiol. 2006;291(3): H1421-1428.

32. Pequignot MO, Provost AC, Salle S, et al The retinal pigment epithelium undergoes massive apoptosis during early differentiation and pigmentation of the optic cup. Mol Vis. 2011;17:989-996.

33. JAX ${ }^{\circledR}$ Hydrocephalus in laboratory mice.Issue 490, Summer 2003 (available from: https://www.jax.org/news-andinsights/2003/july/hydrocephalus-in-laboratory-mice).

34. Kurakula K, Koenis DS, Herzik MA, Jr., et al. Structural and cellular mechanisms of peptidyl-prolyl isomerase Pin1-mediated enhancement of tissue factor gene expression, protein half-life, and pro-coagulant activity. Haematologica. 2018;103(6):10731082.

35. Reinhardt C, Bergentall M, Greiner TU, et al Tissue factor and PAR1 promote microbiota- 
Sex specific TF bleeding phenotype

induced intestinal vascular remodelling. Nature. 2012;483(7391):627-631.

36. Rothmeier AS, Marchese P, Langer F, et al. Tissue factor prothrombotic activity is regulated by integrin-arf6 trafficking. Arterioscler Thromb Vase Biol. 2017;37(7):1323-1331.

37. Connolly AJ, Ishihara H, Kahn ML, Farnese RV Jr, Coughlin SR. Role of the thrombin receptor in development and evidence for a second receptor. Nature. 1996;381(6582): 516-519.

38. Cur J, O'Shea KS, Purkayastha A, Saunders TL, Ginsburg D. Fatal haemorrhage and incomplete block to embryogenesis in mice lacking coagulation factor V. Nature. 1996; 384(6604):66-68.

39. Sun WY, Witte DP, Degen JL, et al. Prothrombin deficiency results in embryonic and neonatal lethality in mice. Proc Natl Acad Sci U S A. 1998;95(13):7597-7602.

40. Damiano BP, Cheung WM, Santulli RJ, et al. Cardiovascular responses mediated by protease-activated receptor-2 (PAR-2) and thrombin receptor (PAR-1) are distinguished in mice deficient in PAR-2 or PAR-1. J Pharmacol Exp There. 1999;288(2):671-678.

41. Parry GC, Mackman N. Mouse embryogenisis requires the tissue factor extracellular domain but not the cytoplasmic domain. J Chin Invest. 2000;105(11):1547-1554.

42. Davis DR, Wilson K, Sam MJ, et al. The development of cardiac fibrosis in low tissue factor mice is gender-dependent and is assocrated with differential regulation of urokinose plasminogen activator. J Mol Cell Cardiol. 2007;42(3):559-571.

43. Van Loo PL, Van Zutphen LF, Baumans V. Male management: coping with aggression problems in male laboratory mice. Lab Anim. 2003;37(4):300-313.

44. Ghio AJ, Ghio C, Basset M. Exerciseinduced pulmonary hemorrhage after runming a marathon. Lung. 2006;184(6):331333.

45. Ko YC, Di MP, On CC. Playing saxophone induced diffuse alveolar hemorrhage: a case report. Ir J Med Sci. 2010;179(1):137-139.

46. Santalo M, Domingo P, Fontcuberta J, Franco M, Nolla J. Diffuse pulmonary hemorrhage associated with anticoagulant therapy. Eur J Respir Dis. 1986;69(2):114-119.

47. Ko LC, Sugihara J, Druger G. First case report of spontaneous pulmonary hemorrage following heparin therapy in acute myocardial infarction. Hawaii Med J. 1996;5 5(5):83-84.

48. Sitges M, Villa FP. Massive pulmonary hemorrhage in a patient treated with a platelet glycoprotein IIb/IIIa inhibitor. Int J Cardiol. 1997;62(3):269-271.

49. Finley TN, Aronow A, Cosentino AM, Golds DW. Occult pulmonary hemorrhage in anticoagulated patients. Am Rev Respir Dis. 1975;112(1):23-29.

50. Rothmeier AS, Lu E, Chakrabarty S, et al. Identification of the integrin-binding site on coagulation factor VIla required for proangiogenic PAR2 signaling. Blood. 2018; 131(6):674-685.

haematological | 2020; 105(10)

2495 